\title{
Interpretação de dados eletromagnéticos para prospecção de água subterrânea no Nordeste Brasileiro
}

\author{
Pedro Henrique da Silva Chibane ${ }^{1}$; Carlos A. Mendonça'; Oderson Souza Filho ${ }^{2}$ \\ 'Departamento de Geofísica IAG/USP, ${ }^{2}$ CPRM - Serviço Geológico do Brasil - Núcleo de Apoio de Curitiba
}

Copyright 2016, SBGf - Sociedade Brasileira de Geofísica

Este texto foi preparado para a apresentação no VII Simpósio Brasileiro de Geofísica Ouro Preto, 25 a 27 de outubro de 2016. Seu conteúdo foi revisado pelo Comitê Técnico do VII SimBGt, mas não necessariamente representa a opinião da SBGf ou de seus associados. É proibida a reprodução total ou parcial deste material para propósitos comerciais sem prévia autorização da SBGf.

\section{Abstract}

Despite being affected by long drought periods, some areas of the Brazilian Northeast has groundwater resources which in principle can serve to public, industrial and agricultural consumption. Proper techniques to improve target evaluation and well location need further developments. To assist groundwater exploration, a frequency domain airborne electromagnetic survey (AEM) was conducted during the PROASNE project, a cooperation between the geological surveys of Brazil (CPRM) and Canada (GSC). Here we interpret part of the PROASNE data (Block Juá, in the State of Ceará) applying a procedure able to identify promising targets for ground investigation and well location.

\section{Introdução}

Levantamentos eletromagnéticos aerotransportados (Airborne Electromagnetics-AEM) foram introduzidos no final da década de 1940 pela empresa Stanmac-McPhar (Fountain, 1998). O sucesso desses levantamentos na exploração mineral, especialmente no distrito de New Brunswick (Canadá) permitiu a aplicação desse método em campanhas de mapeamento geológico (Palacky, 1981), e exploração hidrogeológica (Palacky, 1986), ainda mantendo como ferramenta-exploração mineral (Palacky \& Sena, 1979; Macnae, 1995).

As primeiras aplicações de dados AEM no Brasil são descritas por Palacky \& Sena (1979) e Palacky \& Kadekaru (1979) em projetos para exploração mineral pela então CVRD (Companhia Vale do Rio Doce). Aplicações pioneiras na prospecção de recursos hídricos subterrâneos começaram em 2001, no PROASNE (Projeto Água Subterrânea no Nordeste do Brasil), desenvolvido pela parceria entre o Serviço Geológico do Brasil (CPRM) e o Serviço Geológico do Canadá (GSC). O PROASNE teve como objetivo averiguar a utilidade dos dados AEM na exploração hidrogeológica em terrenos cristalinos do Nordeste Brasileiro, com potencial para ser mais uma ferramenta de suporte às ações de combate aos efeitos da seca. Estudos utilizando dados do PROASNE no Bloco Juá foram desenvolvidos por Souza Filho (2008) e Pinéo et al. (2013), entre outros.

Aplicações recentes de dados AEM em hidrogeologia permitem mapear potenciais litologias e estruturas geológicas aquíferas (Sattel \& Kgotlhang, 2004), determinar a distribuição de salinidade no interior das camadas geológicas localizadas em terrenos sedimentares (Coppa et al., 1998; Meng et al., 2006) e fraturados (Souza Filho et al., 2010), auxiliar locações de rejeito nuclear a partir de modelos hidrogeológicos (Rhén et al., 2007) e mapear o embasamento e zonas fraturadas sob rochas sedimentares (Pedersen et al., 2009).

Como se sabe (Palacky, 1986), a interpretação de dados AEM fornecem a localização e características de alvos condutivos, o que nem sempre fornece uma indicação direta da presença (ou ausência) de água em uma determinada região (Souza Filho et al., 2010). Isso porque alvos condutivos tanto podem indicar meios porosos saturados com água (aquíferos), quanto formações geológicas com minerais mais condutivos (sem água). Surge daí a importância em analisar as propriedades dos alvos determinados, no sentido de identificar aqueles compatíveis com os atributos de um aquífero.

Os objetivos primários deste trabalho são a seleção e interpretação dos alvos com anomalias eletromagnéticas identificadas em uma das áreas-teste do PROASNE, o Bloco Juá, no estado do Ceará. A análise desses resultados será balizada com informação de poços cadastrados no SIAGAS (Sistema de Informações de Água Subterrânea - CPRM) e correlacionada com os arquivos de dados do Bloco Juá fornecidos pela CPRM. Modelos petrofísicos foram aplicados a anomalias selecionadas para quantificação dos parâmetros dos alvos. Uma análise estatística posterior discriminou as anomalias cujas respostas geofísicas e estruturas são favoráveis à captação de água subterrânea.

\section{Levantamentos EM do PROASNE}

Os aerolevantamentos eletromagnéticos e magnéticos pelo projeto PROASNE foram realizados em três estados brasileiros (PROASNE, 2005) pela localização dos principais apoiadores do projeto no Brasil, sendo a CPRM e os grupos em pesquisa das universidades federais do Ceará, Rio Grande do Norte e Pernambuco: Juá (Ceará), Serrinha (Rio Grande do Norte) e Samambaia (Pernambuco). Os dados do Bloco Juá do PROASNE foram obtidos entre os meses de março e maio de 2001 pela LASA Engenharia e Prospecções S.A (LASA, 2001). O levantamento foi executado com helicóptero Esquilo HB-350 equipado com um sistema eletromagnético (Aerodat DSP99) no domínio da frequência (FDEM) com sensor rebocado por um cabo de 30 metros. O sistema opera nas frequências nominais de 900, 4.500 e $33.000 \mathrm{~Hz}$, com bobinas em arranjos coplanar (horizontal) e coaxial (vertical). O espaçamento entre as linhas de voo (E-W) foi de $100 \mathrm{~m}$ e entre linhas de controle (N-S) de $500 \mathrm{~m}$. As etapas de processamento consistiram em remoção de spikes, 
filtragem, determinação do nível de base, seleção das anomalias e cálculo da condutividade aparente.

A interpretação dos dados AEM realizada pela LASA, baseou-se no método discutido por Fraser (1978) e West (1986), o qual determina os parâmetros que descrevem a fonte da anomalia EM por meio de diagramas "fasor" ou de Argand. Os valores das componentes em fase (Ip) e quadratura (Q) da resposta eletromagnética são plotados em um diagrama fasor, correspondente a um modelo de alvo e um arranjo específico de bobinas. Cada arranjo (coplanar e coaxial) possui um diagrama para diferentes modelos geológicos, e atribui diferentes razões entre a altura de voo e a profundidade do topo ( $z$, no modelo de lâmina) ou espessura ( $h$, no modelo de camada de cobertura) do alvo. Este método de análise e interpretação dos alvos foi aplicado apenas aos dados da frequência de 4.500 $\mathrm{Hz}$.

A Figura 1 apresenta a correspondência entre modelos geofísicos e geológicos utilizados na interpretação dos dados EM do Projeto Juá. O modelo D é caracterizado pela condutância e profundidade do topo de uma lâmina condutora. A condutância é dada pelo produto entre a espessura e a condutividade, mostrando que alvos diferentes são capazes de produzir respostas EM equivalentes. $O$ modelo $S$ representa uma cobertura condutiva e é caracterizado por sua condutividade (ou resistividade) e espessura de uma placa horizontal.

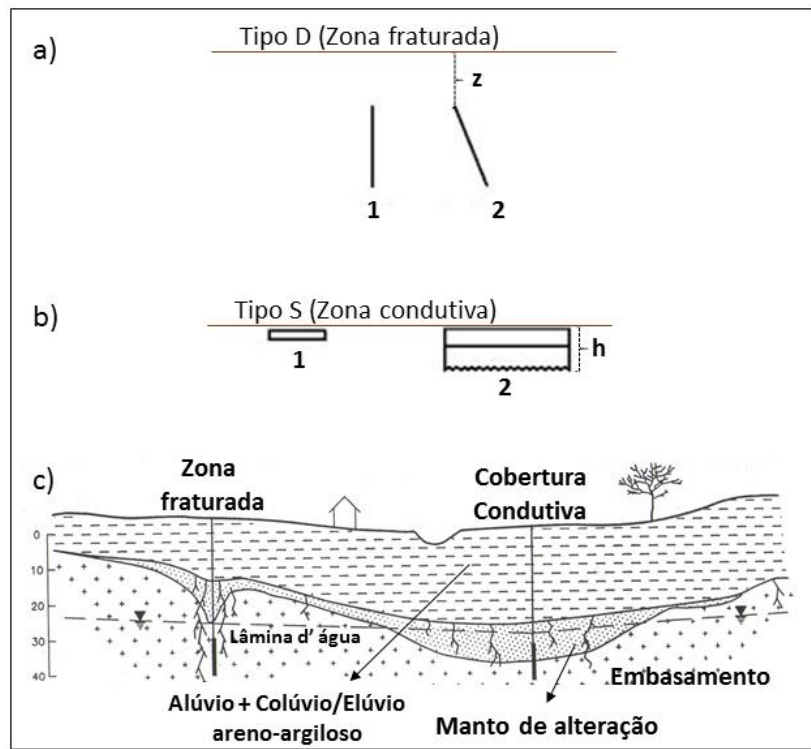

Figura 1: (a) Modelo geofísico do tipo $D$ composto por fraturas vertical e inclinada (1 e 2) com profundidade do topo igual a z; (b) Modelo do tipo $S$ (cobertura condutiva); placa horizontal fina (1); lâmina fina (2) com espessura $h$. (c) Modelo geológico para fraturas e coberturas condutivas. Adaptado de Palacky et al. (1981) e LASA (2001).

Alvos do tipo D e $S$ foram identificados ao longo de cada linha de voo gerando uma lista com as coordenadas de localização, parâmetros dos alvos e classificação das anomalias. Foi possível, assim, avaliar estatisticamente a distribuição das anomalias no Bloco
Juá no intuito de identificar alvos mais favoráveis à captação de água subterrânea.

\section{Localização e Geologia}

A região de estudo está localizada na porção norte do Estado do Ceará (município de Irauçuba, Distrito de Juá), coberta pelo aerolevantamento de $154 \mathrm{~km}^{2}$ do Bloco Juá. A geologia da área (Figura 2) é composta pela unidade Independência do Complexo Ceará, rochas neoproterozoicas do Complexo Tamboril-Santa Quitéria e por coberturas aluvionares recentes. O cadastro do SIAGAS contém informações de 20 poços. A Figura 2 mostra que os poços de maiores vazões ocorrem em depósitos aluvionares ou em sua proximidade. O critério para apresentação dos poços baseou-se em Souza Filho et al. (2007), no qual consideraram-se os intervalos práticos de instalação do equipamento de bombeamento.
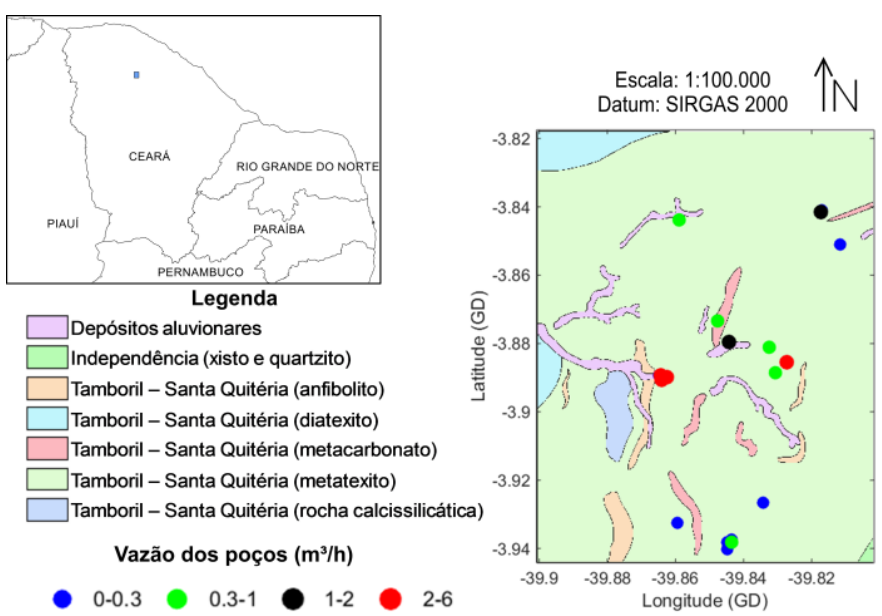

Figura 2: Localização e Geologia do Bloco Juá (CE) e dados de vazão de poços conforme cadastro do SIAGAS. Adaptado de CPRM - GEOBANK, Folha AS.24-Y-D-VIrauçuba.

\section{RESULTADOS}

\section{Condutores tipo D}

A análise dos condutores do tipo $D$ (fraturas) consistiu de rotinas de processamento no MATLAB ${ }^{\odot}$ para plotar o mapa de distribuição dessa fonte na região e histogramas dos parâmetros de condutância e profundidade do topo $(\mathrm{z})$.

O número reduzido deste tipo de alvo mostra que poucas fraturas foram identificadas no levantamento AEM (Figura 3a), mesmo considerando uma região de cristalino com fraturas reconhecidas em campo. Apenas em dois locais observa-se a associação entre alvos do tipo $D$ na vizinhança de dois poços, um com vazão entre 0,3 e $1 \mathrm{~m}^{3} / \mathrm{h}$ e outro entre 1 e $2 \mathrm{~m}^{3} / \mathrm{h}$ (setas amarelas na Figura 3a). A Figura $3 b$ apresenta o histograma de condutâncias para os alvos do tipo $D$, com valores entre 1 e $2 \mathrm{~S}$.

Dos 5023 condutores estudados (tipos D e S), os condutores D representam apenas $0,4 \%$ do total. A justificativa para o baixo valor de fraturas mapeadas 
fundamenta-se na hipótese de que a abertura das fraturas tenha valores pequenos. Considerando que a água doce tenha condutividade de $0,03 \mathrm{~S} / \mathrm{m}$ (ou $30 \Omega . \mathrm{m}$ de resistividade) e que exista 1,43 fraturas $/ \mathrm{m}$ (Boutt et al., 2010), a abertura de fraturas deve ser de $8,5 \mathrm{~cm}$. Na literatura, essa grandeza varia entre 0,05 e $0,20 \mathrm{~mm}$ (Luthi \& Souhaité, 1990). Esta análise sugere que a densidade e a abertura das fraturas, bem como os intervalos de condutividade da água, podem resultar alvos laminares com condutâncias muito baixas, cuja resposta é imperceptível em levantamentos AEM com as especificações normalmente empregadas. Este é um resultado ainda preliminar, que merece investigação mais aprofundada, com base em modelos petrofísicos representativos da região em estudo.

\section{Condutores tipo S}

Condutores do tipo $\mathrm{S}$ podem ser associados a variações de espessura no manto de alteração ou presença de depósitos aluvionares. Coberturas com maior espessura podem, em potencial, apresentar maior armazenagem de água ou de forma decisiva contribuir com a recarga do aquífero fissural subjacente. Feições com resistividade muito baixa, por outro lado, podem representar camadas argilosas ("aquicludes") ou preenchidas com água salgada (baixa qualidade da água). A Figura 4a apresenta a distribuição dos condutores S no Bloco Juá (CE). Mesmo excluindo alvos com valores espúrios de condutividade (valores infinitos), o número de condutores é ainda elevado (4281), impedindo estabelecer alguma associação desses alvos com os poços mais produtivos da região.

Para selecionar alvos mais favoráveis, utilizaramse as estimativas de condutividade e espessura da lista de alvos para determinar um parâmetro, $\mathrm{T}$, igual ao produto da espessura pela resistividade (ou espessura dividida pela condutividade). Essa grandeza é denominada como resistência transversal, ou parâmetro T de Dar Zarrouk (Batayneh, 2013), muito utilizado em estudos hidrogeológicos em meio sedimentar para caracterizar camadas aquíferas ou atuando como aquicludes. No contexto do Projeto Juá, foi suposto que valores mais altos de resistência transversal podem indicar coberturas mais espessas (maior armazenagem) e com águas mais resistivas (menor salinidade). Calculou-se, assim, a resistividade e a resistência transversal para todos os alvos do tipo $\mathrm{S}$ com a finalidade de selecionar os condutores com resistividade igual ou acima de $30 \Omega . m$ (supostamente aquíferos com água doce) e espessas (resistência transversal maior que de $300 \Omega \cdot \mathrm{m}^{2}$ ), conforme mostra a Figura 5. A Figura $4 \mathrm{~b}$ mostra os modelos tipo $S$ que atendem 0 critério estabelecido. Verifica-se que existem diversas fontes anômalas, mas sem relação evidente com terrenos aluvionares. Porém, no Norte da área se destaca um aluvião onde um poço mostrou vazão entre 0,3 e $1 \mathrm{~m}^{2} / \mathrm{h}$ (Figura 4b), coincidente com a distribuição de fraturas em um dos poços. Em outros locais, os alvos favoráveis aparecem em linhas contíguas, porém em locais sem dados de poço. A princípio, os locais com alvos promissores segundo o parâmetro de Dar Zarrouk deveriam ser investigados em campo, utilizando perfis geofísicos de resistividade para verificar se, de fato, o manto de alteração é mais espesso.

\section{Discussão e Conclusões}

Os resultados apresentados na Figura 3 mostram o número reduzido de condutores do tipo D (fraturas), o que pode ser indicativo de fraturas com baixa condutância elétrica. A condutância depende de muitos parâmetros que caracterizam uma zona fraturada, tais como: densidade e abertura de fraturas, condutividade da água de poro, espessura da zona fissurada, entre outros. Uma possibilidade é que as fraturas da região não apresentam atributos que permitam sua detecção, ao menos com base nas especificações do levantamento efetuado. Essa questão aponta para a necessidade de se obter modelos petrofísicos oriundos de investigações de campo, especialmente com o uso de perfilagem geofísica para caracterizar o aquífero fissural.

Os resultados na Figura 4 mostram a importância em selecionar alvos representativos de coberturas condutivas conforme critérios objetivos, compatíveis com propriedades esperadas para os aquíferos da região. $\mathrm{O}$ parâmetro proposto no presente estudo é o parâmetro T de Dar Zarrouk, que pode ser inferido a partir dos dados AEM (considerando modelos com uma camada apenas). Não necessariamente a locação de poços deve ser realizada apenas com base neste parâmetro, mas acredita-se que ele possa ser usado como referência para orientar estudos de detalhe em condições de campo. Perfis de resistividade elétrica ou de sísmica de refração podem, por exemplo, verificar locais nos quais o espessamento das coberturas superficiais foi inferido, só então definindo alvos mais precisos para a locação de poços. Para condutores do tipo $D$ (fraturas) recomendase investigar o local próximo a um poço com vazão entre 0,3 e $1 \mathrm{~m}^{3} / \mathrm{h}$ e com alvos compatíveis com fraturas (Figura 3b).

\section{Agradecimentos}

Ao convênio CPRM/IAG №25256 por ceder os dados eletromagnéticos referente ao estudo no Bloco Juá (CE). À SBGf pelo financiamento na forma de bolsa de Iniciação Científica e pela oportunidade de realizar uma pesquisa que pode agregar conhecimento e trazer benefícios para a população.

\section{Referências}

Batayneh, A. T. 2013. The estimation and significance of Dar-Zarrouk parameters in the exploration of quality affecting the Gulf of Aqaba coastal aquifer systems. Journal of Coastal Conservation, 17(3), 623635.

Boutt, D. F., Diggins, P. \& Mabee, S. 2010. A field study (Massachusetts, USA) of the factors controlling the depth of groundwater flow systems in crystalline fractured-rock terrain. Hydrogeology Journal, 18(8), 1839-1854.

Coppa, I., Woodgate, P. \& Webb, A. 1998. Improving the management of dryland salinity in Australia through the national airborne geophysics project. Exploration geophysics, 29(1/2), 230-233. 
Fountain, D. 1998. Airborne electromagnetic systems-50 years of development. Exploration Geophysics, 29(1/2), 1-11.

Fraser, D. C. 1978. Resistivity mapping with an airborne multicoil electromagnetic system. Geophysics, 43(1), 144172.

LASA (Engenharia e Prospecções S.A.). 2001. Projeto Aerogeofísico Água Subterrânea no Nordeste do Brasil Blocos Juá (CE), Samambaia (PE) e Serrinha $(R N)$ Relatório Final do Levantamento e Processamento dos Dados Magnetométricos e Eletromagnetométricos e Seleção das Anomalias Eletromagnéticas. Texto Técnico, Relatório Final, V. 1, 82 p, Rio de Janeiro.

Luthi, S. M., \& Souhaité, P. 1990. Fracture apertures from electrical borehole scans. Geophysics, 55(7), 821-833.

Macnae, J. 1995. Applications of geophysics for the detection and exploration of kimberlites and lamproites. Journal of Geochemical Exploration, 53(1), 213-243.

Meng, Q., Hu, H., \& Yu, Q. 2006. The application of an airborne electromagnetic system in groundwater resource and salinization studies in Jilin, China. Journal of Environmental \& Engineering Geophysics, 11(2), 103109.

Palacky, G. J. \& Sena, F. O. 1979. Conductor identification in tropical terrains - Case histories from the Itapicuru greenstone belt, Bahia, Brazil. Geophysics, 44(12), 1941-1962.

Palacky, G. J. \& Kadekaru, K. 1979. Effect of tropical weathering on electrical and electromagnetic measurements. Geophysics, 44(1), 69-88.

Palacky, G. J. 1981. The airborne electromagnetic method as a tool of geological mapping. Geophysical Prospecting, 29(1), 60-88.

Palacky, G. J., Ritsema, I. L. \& Jong, S. D. 1981. Electromagnetic Prospecting for Groundwater in Precambrian Terrains in the Republic of Upper VOLTA*. Geophysical Prospecting, 29(6), 932-955.

Palacky, G. J. 1986. Geological background to resistivity mapping. Ed., Palacky, GJ, Airborne resistivity mapping, GSC Paper, 86(22), 19-27.
Pedersen, L. B., Persson, L., Bastani, M. \& Byström, S. 2009. Airborne VLF measurements and mapping of ground conductivity in Sweden. Journal of applied geophysics, 67(3), 250-258.

Pinéo, T. R. G., Branco, R. M. G. C., da Cunha, L. S. \& Souza, M. L. 2013. Airborne and ground geophysics applied to groundwater prospection in hard rocks in Irauçuba, Ceará state, Brazil. Revista Brasileira de Geofísica,31(4), 699-709.

PROASNE. 2005. Northeastern Brazil Groundwater Project PROASNE (2000-2004) closing report; Org. Maurice, Y T. Geological Survey of Canada, Open File 5440, 2007; viii, 65 (+app.) pages; 1 CD-ROM, doi:10.4095/223385.

Rhén, I., Thunehed, H., Triumf, C. A., Follin, S., Hartley, L., Hermansson, J. \& Wahlgren, C. H. 2007. Development of a hydrogeological model description of intrusive rock at different investigation scales: an example from southeastern Sweden. Hydrogeology Journal, 15(1), 47-69.

Sattel, D. \& Kgotlhang, L. 2004. Groundwater exploration with AEM in the Boteti area, Botswana. Exploration Geophysics, 35(2), 147-156.

Souza Filho, O. A., Silva, A. M., Perrotta, M. M. \& McCafferty, A. 2007. PROPRIEDADES GEOFÍSICAS DE LINEAMENTOS ASSOCIADOS ÀS VAZÕES DE POÇOS EM AQÜÍFEROS CRISTALINOS NA REGIÃO DE JUÁ, CEARÁ, BRASIL. Águas Subterrâneas, (1).

Souza Filho, O.A. 2008. Dados aerogeofísicos e geológicos aplicados à seleção de áreas favoráveis para água subterrânea no domínio cristalino do Ceará, Brasil. Tese de Doutorado, Universidade de Campinas, São Paulo.

Souza Filho, O. A., Silva, A. M., Remacre, A. Z., Sancevero, S. S., McCafferty, A. E. \& Perrotta, M. M. 2010. Using helicopter electromagnetic data to predict groundwater quality in fractured crystalline bedrock in a semi-arid region, Northeast Brazil. Hydrogeology journal, 18(4), 905-916.

West, G. F. 1986. Modelling of airborne electromagnetic response: present capabilities and future expectations. Ed., Palacky, GJ, Airborne resistivity mapping, GSC Paper, 86(22), 29-38. 

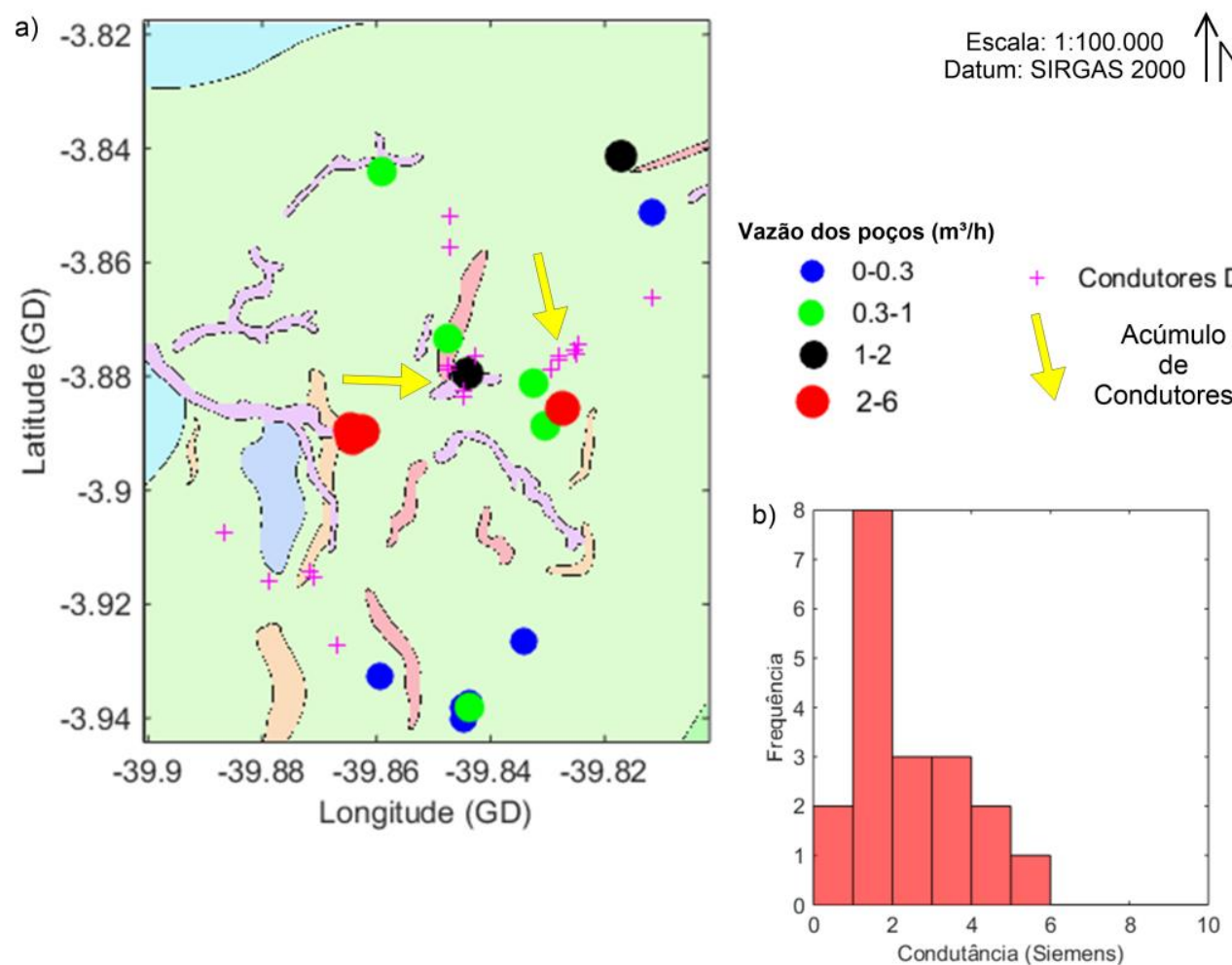

Figura 3: (a) Distribuição das fraturas (cruz em rosa) e poços cadastrados no SIAGAS. As setas amarelas indicam acúmulo de condutores, indicando possível relação com os poços próximos; (b) Histograma de Condutância (Siemens) para a anomalia do tipo D.
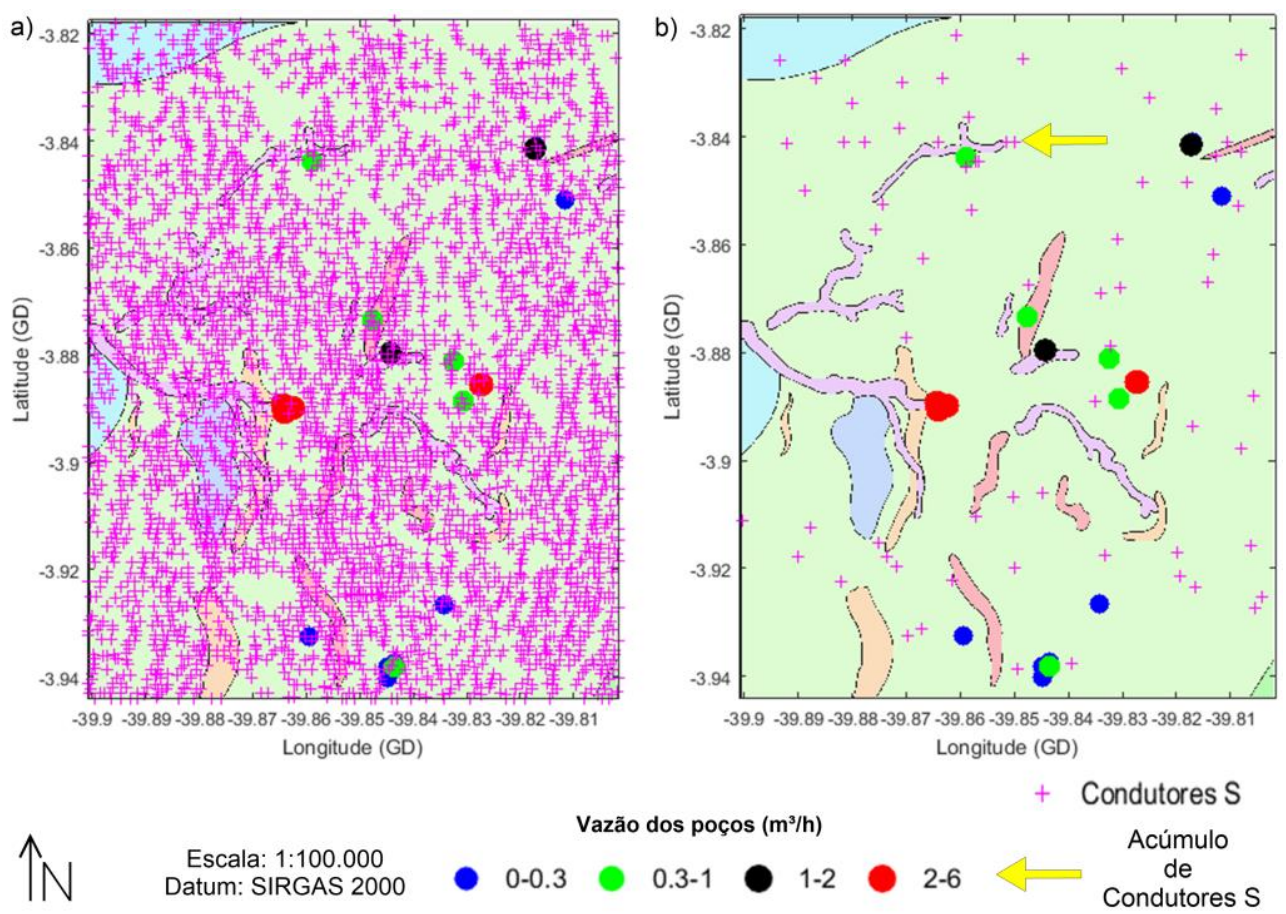

+ Condutores S

Acúmulo

$$
\text { de }
$$

Figura 4: (a) Distribuição de condutores do tipo $S$ (cruz em rosa) e os poços SIAGAS contidos no Bloco Juá (CE). A seta amarela indica acúmulo de condutores $S$ na região; (b) Anomalias do tipo $S$ que possuem resistividade maior que $30 \Omega . m$ e resistência transversal unitária $T$ maior que $300 \Omega \mathrm{m}^{2}$. Códigos de processamento e preparação de figuras 
a)

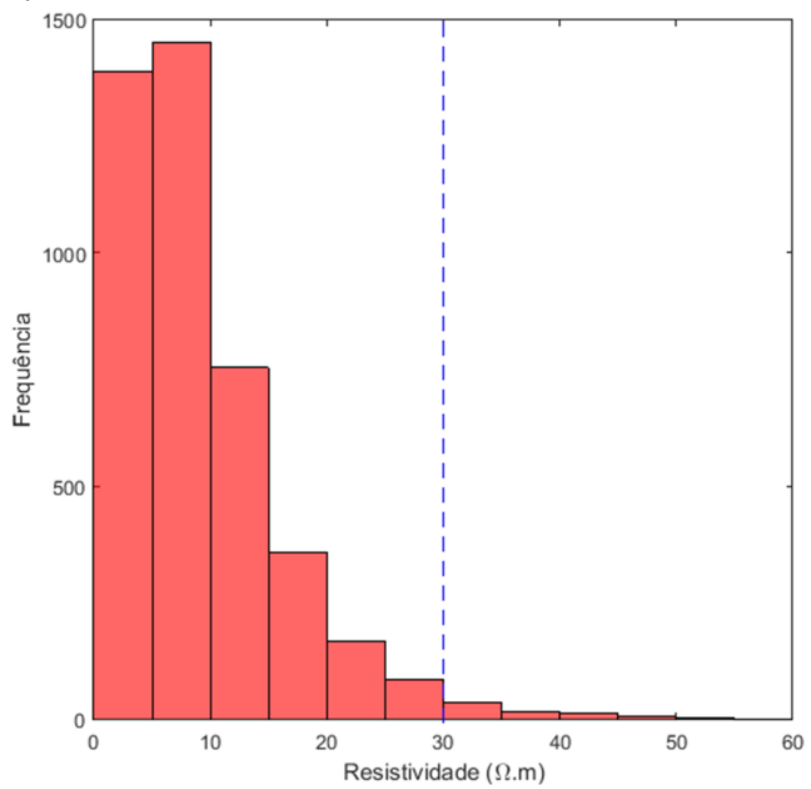

b)

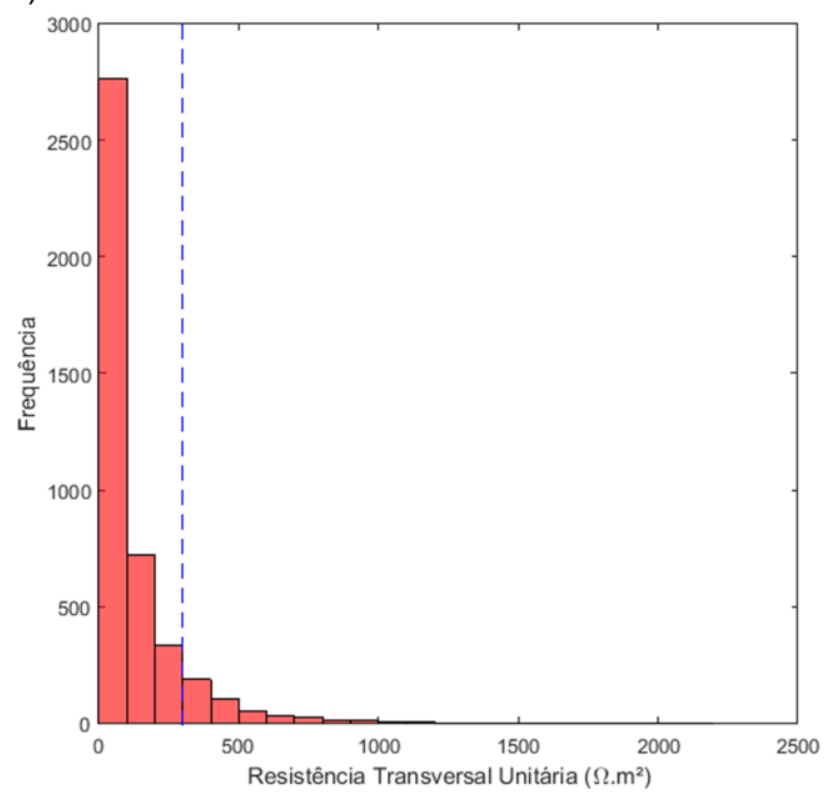

Figura 5: (a) Histograma de resistividade ( $\Omega . m$ ), onde a linha tracejada (azul) representa o valor de corte no qual valores iguais ou superiores a 30 S.m foram considerados ideais para água doce; (b) Histograma do parâmetro T de Dar Zarrouk (Resistência transversal unitária), no qual valores iguais ou acima de $300 \Omega . m^{2}$ (linha tracejada em azul) foram considerados ideais para água doce. 